\title{
A numerical inversion method for CCD side-scatter lidar
}

\author{
Zongming $\mathrm{Tao}^{* 1,2}$, Dong $\mathrm{Liu}^{2}$, Chenbo Xie ${ }^{2}$, Xiaomin \\ $\mathrm{Ma}^{1}$, Xiangqian Meng ${ }^{2}$, Shunxing $\mathrm{Hu}^{2}$, Yingjian Wang ${ }^{2}$ \\ (1 New Star Institute of Applied Technology, \\ Hefei, Anhui, China, 230031)
}

\begin{abstract}
Using a CCD as the detector, side-scatter lidar has great potential application in the near range atmospheric detection. Side-scatter lidar equation is different from backscatter lidar in terms of atmospheric transmittance and atmospheric scatter coefficient. A new inversion method is proposed for side-scatter lidar retrievals, and numerical simulation is used to analyze the sensitivity to aerosol backscatter coefficient at the reference point. The results presented in this paper show that this method is reasonable and feasible.
\end{abstract}

Key words-Atmospheric optics; Aerosol; Side-scatter lidar; Inversion

\section{INTRODUCTION}

Aerosols play a strong role in global climate change both directly, by affecting Earth's radiation balance, and indirectly, by affecting cloud properties[1]. Understanding their contribution to climate change requires aerosol altitude profile information[2]. As aerosol types, chemstry, concentrations are highly variable and strongly altitude dependent, measurements of aerosol properties as a function of altitude are especially important. The majority of aerosols exsit in the lower few kilometers in near surface. This aerosol-rich layer is generally called the atmospheric (or planetary) boundary layer. Remote sensing techniques have been applied to aerosol detection by use of a variety of instruments. Sun photometers provide total collumn aerosol content, indicative of the aerosol properties integrated through the entire altitude range of the atmosphere. Backscatter lidars are capable of providing range-resolved atmospheric measurements continuously, making backscatter lidars valuable tools in the remote sensing of atmospheric aerosols[3]. But the backscatter lidar system has a shortage in the lower hundreds of meters because of the overlap function caused by the configuration of the transmitter divergence and receiver field of view at ranges close to the instrument[4]. This limits the backscatter lidar to apply to near range field.

Applied a charge coupled device (CCD) camera detector, a side-scatter lidar, in which the detector is spatially separated from the transmitter by a certain distance, is different to backscatter lidar, in which the transmitter and detector are collocated. The side-scatter lidar solved the overlap problem in backscatter lidar, and is especially suitable to apply to measure aerosol in near range[5-6]. But there is a new difficult problem that is how to solve the side-scatter lidar equation.
(2 Key laboratory of Atmospheric Composition and Optical

Radiation, Chinese Academy of Sciences, Hefei, Anhui, 230031 China)

*E-mail: zmtao@aiofm.ac.cn

\section{TECHNIQUE}

The diagram of side-backsactter lidar is shown in Fig. 1. For side-scatter lidar configuration, the lidar equation can be described as[5]

$$
P(z, \theta)=\frac{P_{0} K A}{D} \beta(z, \theta) T_{t} T_{r} d \theta,
$$

Where $P(z, \theta)$ is the received power at height $\mathrm{z}$ and scattering angle $\theta$ by a pixel, $K$ is a calibration constant representing the system optical efficiency and $\mathrm{A}$ is the effective collecting area of the optics, and $T_{t}$ and $T_{r}$ are the total (aerosol and molecular) atmospheric transmittance from the laser to altitude $\mathrm{z}$ and from altitude $\mathrm{z}$ along the slant path to the CCD receiver, respectively. $\beta(z, \theta)$ is the total atmospheric sidescattering coefficient. Because of $\theta=\pi / 2+\gamma, d \theta$ (equal to $d \gamma$ ) is one pixel field of view.

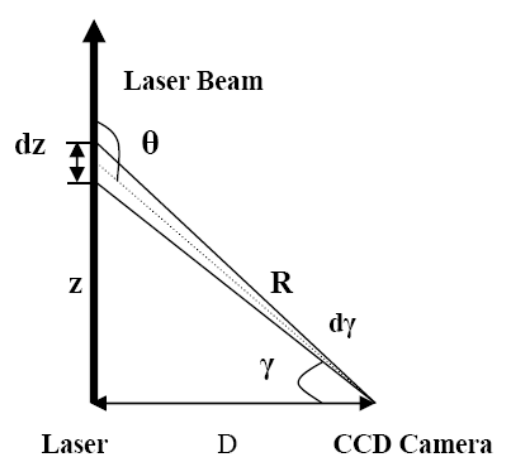

Fig. 1. Side-scatter lidar diagram

For CCD receiver, side-backscatter lidar signals record by different pixels. From geometry, the spacial resolution $d z$ is expressed as

$$
d z=\frac{R^{2}}{D} d \theta .
$$

For CCD receiver, the pixel field of view $d \theta$ is approximate constant, so the spacial resolution $d z$ increases with slant distance $R$.

By comparison equations (1) and (2) with backscatter lidar corresponding equations, side-scatter lidar has two main 
difference aspects with backscatter lidar, one is spacial resolution is not constant, the another is received power is not $1 / R^{2}$ dependence. The cancellation of the $1 / R^{2}$ dependence has beneficial consequences for the dynamic range of the sidescatter lidar system. For short range detection, such as atmospheric boundary layer, the spacial resolution of sidescatter lidar still are good in suitable optics design.

\section{METHOD}

To backscatter lidar equation, Fernald provided an interactive solution[7]. But to side-scatter lidar equation, Fernald's inversion method is not suitable, because of atmospheric transmittance difference and adoption of the aerosol phase function. The side-scattering coefficient $\beta(\theta)$ and the total scattering coefficient $\beta_{s}$ of aerosol have the relationship as

$$
\beta(\theta)=f(\theta) \beta_{s},
$$

Where $f(\theta)$ is phase function of aerosol. If backsacttering coefficient is known, the side-scattering coefficient $\beta(\theta)$ can be expressed as

$$
\beta(\theta)=\frac{\beta(\pi)}{f(\pi)} f(\theta) .
$$

If we consider the atmosphere is homogenous in the horizontal, side-scatter lidar equation can be rewritten as

$$
\begin{aligned}
& P(z, \theta)=\frac{P_{0} K A}{D}\left(\frac{\beta_{1}(z, \pi)}{f_{1}(\pi)} f_{1}(\theta)+\frac{\beta_{2}(z, \pi)}{f_{2}(\pi)} f_{2}(\theta)\right) \bullet \\
& \exp -\left(\int_{0}^{z}\left(\alpha_{1}\left(z^{\prime}\right)+\alpha_{2}\left(z^{\prime}\right)\right) d z^{\prime}+\int_{0}^{z / \cos (\pi-\theta)}\left(\alpha_{1}\left(z^{\prime}\right)+\alpha_{2}\left(z^{\prime}\right)\right) d z^{\prime}\right) d \theta
\end{aligned}
$$

where $\alpha(z)$ is extinction coefficient, subscripts " 1 " and " 2 " indicate aerosol and molecule respectively.

Using optical depth $\tau=\int_{0}^{z}\left(\alpha_{1}\left(z^{\prime}\right)+\alpha_{2}\left(z^{\prime}\right)\right) d z^{\prime}$, equation (5) becomes as

$$
\begin{aligned}
& P(z, \theta)=\frac{P_{0} K A}{D}\left(\frac{\beta_{1}(z, \pi)}{f_{1}(\pi)} f_{1}(\theta)+\frac{\beta_{2}(z, \pi)}{f_{2}(\pi)} f_{2}(\theta)\right) \bullet . \\
& \exp (-\tau-\tau / \cos (\pi-\theta)) d \theta
\end{aligned}
$$

At reference point $z_{c}$, aerosol backscatter coefficient $\beta_{1}\left(z_{c}, \pi\right)$ is known, we get

$$
\begin{aligned}
& P\left(z_{c}, \theta\right)=\frac{P_{0} K A}{D}\left(\frac{\beta_{1}\left(z_{c}, \pi\right)}{f_{1}(\pi)} f_{1}\left(\theta_{c}\right)+\frac{\beta_{2}\left(z_{c}, \pi\right)}{f_{2}(\pi)} f_{2}\left(\theta_{c}\right)\right) \bullet . \\
& \exp \left(-\tau_{c}-\tau_{c} / \cos \left(\pi-\theta_{c}\right)\right) d \theta_{c}
\end{aligned}
$$

We define $\mathrm{C}$ as

$$
C=\frac{P_{0} K A}{D} \exp \left(-\tau_{c}-\tau_{c} / \cos \left(\pi-\theta_{c}\right)\right)
$$

In practice, we determine $\mathrm{C}$ using

$$
C=\frac{P\left(z_{c}, \theta_{c}\right)}{\left(\frac{\beta_{1}\left(z_{c}, \pi\right)}{f_{1}(\pi)} f_{1}\left(\theta_{c}\right)+\frac{\beta_{2}\left(z_{c}, \pi\right)}{f_{2}(\pi)} f_{2}\left(\theta_{c}\right)\right) d \theta_{c}} .
$$

The attenuated side-scatter $\beta^{\prime}(z, \theta)$, can then be computed

$$
\begin{gathered}
\beta^{\prime}(z, \theta)=\frac{P(z, \theta)}{C}, \\
\beta^{\prime}(z, \theta)=\left(\frac{\beta_{1}(z, \pi)}{f_{1}(\pi)} f_{1}(\theta)+\frac{\beta_{2}(z, \pi)}{f_{2}(\pi)} f_{2}(\theta)\right) \bullet
\end{gathered}
$$

$\exp \left[-\left(\Delta \tau+\tau_{c}\left(1 / \cos (\pi-\theta)-1 / \cos \left(\pi-\theta_{c}\right)\right)+\Delta \tau / \cos (\pi-\theta)\right)\right] d \theta$

Where $\Delta \tau=\int_{z_{c}}^{z}\left(\alpha_{1}\left(z^{\prime}\right)+\alpha_{2}\left(z^{\prime}\right)\right) d z^{\prime}$. When $z<z_{c}$, $\Delta \tau<0$; otherwise $\Delta \tau>0$.

Known aerosol phase function, extinction to backscatter ratio and aerosol backscatter coefficient at reference point, aerosol extinction or backscatter coefficient can be retrieved from $z_{c}$ to consecutive points one after one as the followed steps.

Step 1 using equation (9) and aerosol backscatter coefficient at reference point $z_{c}$, get the constant $\mathrm{C}$.

Step 2 using equation (10a), get the attenuated side-scatter $\beta^{\prime}(z, \theta)$.

Step 3 giving the optical depth $\tau_{c}$, supposing the aerosol backscatter value $\beta_{1, s}$ changed in a reasonable range, compute $\Delta \tau=\beta_{1, s} S 1 \Delta z$ (S1 is aerosol extinction to backscatter ratio ), then using equation (10b) to get the supposed attenuated sidescatter, $\beta_{s}^{\prime}(z+d z, \theta+d \theta)$.

Step 4 compute the difference $\delta=\beta_{s}^{\prime}(z+d z, \theta+d \theta)-\beta^{\prime}(z+d z, \theta+d \theta)$,when $\delta$ is minimum, the corresponding backsacter coefficient value $\beta_{1, s}$ is the retrieved value.

Step 5 from the retrieved aerosol extinction coefficient profile, compute the supposed optical $\operatorname{depth} \tau_{c, s}=\int_{0}^{z_{c}}\left(\alpha_{1}\left(z^{\prime}\right)+\alpha_{2}\left(z^{\prime}\right)\right) d z^{\prime}$.

Step 6 if $\tau_{c}$ is not equal to $\tau_{c, s}$, leting $\tau_{c}=\tau_{c, s}$, repeat step 3 and 5 , until $\left|\left(\tau_{c}-\tau_{c, s}\right) / \tau_{c, s}\right| \leq 0.1 \%$.

\section{SENSITIVITY ANALYSIS AND CONCLUSION}

In order to check the above inversion method, numerical simulation is investigated. Supposing a simulation aerosol extinction profile, aerosol phase function, and extinction to backscatter ratio, from equation (5), we can get the simulation side-scatter lidar signals. Taking the altitude $4.24 \mathrm{~km}$ as reference point, and letting the aerosol backscatter coefficient having a relative error at as reference point, using our inversion method, the retrieved aerosol extinction profile is shown in Fig. 2 . The grey line is the supposed aerosol extinction profile and the black solid line is retrieved one with a 5\% relative error for aerosol backscatter coefficient at reference point, the black dot line is retrieved one with a $10 \%$ relative error for aerosol backscatter coefficient at reference point. From Fig. 2, we see that in altitude $2.5 \mathrm{~km}$ to $4.5 \mathrm{~km}$ range, the retrieved aerosol extinction coefficients are sensitive to backscatter coefficient 
error at reference point. But for atmospheric boundary layer 0 to $2.5 \mathrm{~km}$, the retrieved aerosol extinction coefficients are not changed with backscatter coefficient deviation at reference point. This means that this inversion method is convergence in backward direction computation. So this simulation test indicates that this method is reasonable and feasible.

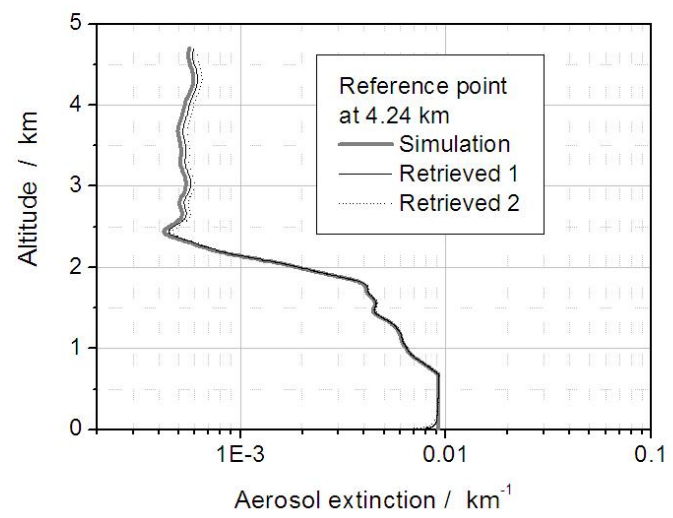

Fig. 2. Simulation test for inversion method.

As the extension of our technique, aerosol phase function can be measured by side-scatter lidar combined with other instrument[8]. In this case, side-scatter lidar emits laser in horizontal direction. If the aerosol extinction and backscatter coefficients are obtained, the aerosol phase function can be retrieved by our inversion method also.
This work was supported by the National Natural Science Foundation of China under Grant No. 41175021, No. 41075016 and No. 41005014.

\section{REFFRENCES}

[1] R. J. Charlson, S. E. Schwartz, J. M. Hales, R. D. Cess, J. A. Coakley, J. E. Hansen, and D. J. Hofmann, "Climate forcing by anthropogenic aerosols," Science 255, 423-430, 1992.

[2] A. Ansmann, D. Althausen, U. Wandinger, K. Franke, D. Miiller, F. Wagner, and J. Heintzenberg, "Vertical profiling of the Indian aerosol plume with six-wavelength lidar during INDOEX: a first case study, " Geophys. Res. Lett. 27, 963-966, 2000.

[3] C. Weitkamp, "Lidar:Range-Resolved Optical Remote Sensing of the Atmosphere," Springer, New York, 2005.

[4] T. Halldorsson and J. Langerhoic, "Geometrical form factors for the lidar function," Appl. Opt. 17, 240-244, 1978.

[5] J. E. Barnes, S. Bronner, R. Beck, and N. C. Parikh, "Boundary layer scattering measurements with a charge-coupled device camera lidar," Appl. Opt. 42, 2647-2652, 2003.

[6] J. E. Barnes, N. C. Parikh Sharma, and T. B. Kaplan, "Atmospheric aerosol profiling with a bistatic imaging lidar system," Appl. Opt. 46, 2922-2929, 2007.

[7] F. G. Fernald, "Analysis of atmospheric lidar observation: some comments," Appl. Opt. 23, 652-653, 1984.

[8] Meng Xiangqian, Hu Shunxing, Wang Yingjian, Hu Huanling, "Aerosol scattering phase functon and visibility based on charge coupled device, ” Acta Optica Sinica, 32, 0901001-1, 2012. 